\title{
Extraction of Ridges-Valleys for Feature-Preserving Simplification of Polygonal Models
}

\author{
Soo-Kyun Kim ${ }^{1}$, Sun-Jeong Kim² , and Chang-Hun Kim ${ }^{3, \star}$ \\ ${ }^{1}$ Samsung Electronics \\ nicesk@gmail.com \\ ${ }^{2}$ Div. of Info. Eng. and Telecom, Hallym University \\ sunkim@hallym.ac.kr \\ ${ }^{3}$ Dept. of Computer Science and Engineering, Korea University \\ chkim@korea.ac.kr \\ Tel.: +82-2-3290-3199; Fax: +82-2-953-0771
}

\begin{abstract}
We propose a new method for simplifying polygonal models while retaining salient features. We extract ridge and valley features defined by means of curvature derivatives on the model surface. We combine this extraction with a simplification technique so as to construct coarse models with features preserved. Experimental results have better quality and smaller geometric error than these of previous methods.
\end{abstract}

\section{Introduction}

Over the last decade, many simplification algorithms have been developed that transform a 3D polygonal model into a simpler version, while preserving its original shape and appearance. Although existing techniques produce plausible results in many cases, the semantic or high-level meaning of the detail of models are often disregarded, because perception of these features is subjective. For example, in the dragon model of Figure 3. regions such as the sharp backbone can be semantically crucial, although they have which will affect small geometric errors any attempt to simplify them.

Some approaches [10, 13 allow users to guide the simplification of semantic features; but such methods require users to select a threshold, which cannot be found intuitively, and users have to discover the threshold value which allow a reasonable set of semantic features to be found. This tedious process requires a lot of skill and time.

We believe that it is possible to get a better result by using a new featureextraction technique to find semantic features in dense polygonal models. This technique identifies well-connected ridges and valleys that represent salient features. Then we compute a simplifying criterion based on quadric and feature quadric error. Finally, a new quadric error metric is generated by combining these two metrics.

\footnotetext{
* The Corresponding author.
} 


\section{Previous Work}

Many successful mesh-simplification algorithms 14, 16 are based on iterative edge contraction [4,5]. They allow vertices to be moved so as to be controlled to preserve the shape or appearance of the original model. Garland [5] and Hoppe [6] use an extended error metric that combines geometric and surface attributes such as normals, colors and texture coordinates. This extended method produces a more accurate and a higher-quality mesh than geometric information alone. Olano et al. 3. use a texture error measurement algorithm to control simplification.

Kho and Garland [10] introduced a user-guided simplification technique and Pojar et al. [13 have presented a method for the user-controlled creation of multiresolution meshes. But these methods still require the user to select regions of geometry to be preserved.

Avioding the need for user-guided features, Watanabe and Belyaev [17] introduced a method for the stable detection of salient curvature extrema on surfaces approximated by dense meshes, which preserves triangles of significant curvature using the Garland-Heckbert method 4. Hubeli et al. 7] proposed a method to find feature edges in $3 \mathrm{D}$ meshes that gives weights to edges based on the difference between two normals, and then fits polynomials to the lines of intersection between a parameter plane and a mesh. A drawback of this method is that users must select a threshold value that cannot be found intuitively. Besides, neither method eliminates the necessity of extracting feature points from the 3D mesh. Yoshizawa et al. [19] have extracted feature lines by using local polynomial fitting of triangulated meshes, which they combined with a quadric-based simplification technique. Kim et al. 8 showed that discrete curvature can be a good criterion for a simplification that preserves the shape of an original model. Walter and Healey [18] have proposed applying feature-preserving mesh simplification to a multidimensional dataset.

\section{Feature Extraction}

Our approach to finding line-type features is based on the method of extracting ridges and valleys due to Kim et al. [9. As a foundation for feature extraction, we will briefly discuss the basics of finding ridge and valley lines, which are curves on a surface formed by the set of loci of points at which the principal curvature has an extremum along its curvature line.

Consider a dense smooth oriented surface. Let $\kappa_{\max }$ and $\kappa_{\min }$ be the maximal and minimal curvatures respectively $\left(\kappa_{\max }>\kappa_{\min }\right)$, with $\mathbf{t}_{\max }$ and $\mathbf{t}_{\min }$ as the corresponding principal directions. The derivatives of $\kappa_{\max }$ and $\kappa_{\min }$ in directions $\mathbf{t}_{\max }$ and $\mathbf{t}_{\min }$ are given by the zero-crossing of $e_{\max }=\nabla \kappa_{\max } \cdot \mathbf{t}_{\max }$ and $e_{\min }=\nabla \kappa_{\min } \cdot \mathbf{t}_{\min }$. Note that we exclude umbilical points, at which $\kappa_{\max }$ $=\kappa_{\min }$. The extrema of principal curvatures along their curvature directions can be used to define ridges and valleys. The ridges are given by

$$
\nabla e_{\max } \cdot \mathbf{t}_{\max }<0, \quad \kappa_{\max }>\left|\kappa_{\min }\right|
$$


and valleys by

$$
\nabla e_{\min } \cdot \mathbf{t}_{\min }>0, \quad \kappa_{\min }<-\left|\kappa_{\max }\right| \cdot
$$

We aim to detect ridges and valleys from an input polygonal mesh, not a smooth surface. First we estimate the curvatures and their derivatives directly from the mesh by employing a local MLS (moving-least-squares) approximation technique to fit the discrete surface. MLS approximations to surfaces usually involve two phases: determining the local tangent plane and then computing a local polynomial over that plane. But our modified technique uses a substitute for the tangent plane, with a corresponding reduction in time-complexity. We quickly estimate curvatures and their derivatives at each vertex using a local polynomial that moves over the plane. Then, we detect ridge and valley vertices by checking for zero-crossings of the extrema of principal curvatures at mesh edges. Finally, the ridge and valley vertices that we have found are connected in the direction of principal curvature.

We shall omit specific references to valleys in this paper, since the methods for detecting ridges and valleys are essentially duals of one another.

\section{New Quadric Error Metric}

It is hard to measure an accurate feature edge on a highly curved surface using only a quadric error metric. We therefore introduce a new quadric error metric (NQEM), which is a combination of a basic error metric with a feature quadric error metric. It preserves features which are removed by a basic error metric.

\subsection{Basic Quadric Error Metric}

The quadric error metric uses a quadric form [4] as its distance error metric which is computed as the weighted sum of squared distances. On each face $f$ of the original mesh, a quadric $Q^{f}(\mathbf{p})$ is defined, which equal to the squared distance from a point $\mathbf{p} \in \mathbf{R}^{3}$ to the plane containing the face $f$. Each vertex $v$ of the original mesh is assigned the sum of quadrics on its adjacent faces weighted by face area:

$$
Q^{v}(\mathbf{p})=\sum_{v \in f} \operatorname{area}(f) \cdot Q^{f}(\mathbf{p}) .
$$

Let us now derive $Q^{f}(\mathbf{p})$ for a given face $f=\left(v_{1}, v_{2}, v_{3}\right)$. The signed distance of $\mathbf{p}$ to the plane containing $f$ is $\boldsymbol{n}^{T} \mathbf{p}+d$, where the face normal $\boldsymbol{n}=\left(\mathbf{p}_{2}-\mathbf{p}_{1}\right) \times$ $\left(\mathbf{p}_{3}-\mathbf{p}_{1}\right) /\left\|\left(\mathbf{p}_{2}-\mathbf{p}_{1}\right) \times\left(\mathbf{p}_{3}-\mathbf{p}_{1}\right)\right\|$ and the scalar $d=-\boldsymbol{n}^{T} \mathbf{p}_{1}$. These parameters can be obtained by solving the linear system,

$$
\left(\begin{array}{ll}
\mathbf{p}_{1}^{T} & 1 \\
\mathbf{p}_{2}^{T} & 1 \\
\mathbf{p}_{3}^{T} & 1
\end{array}\right)\left(\begin{array}{l}
\boldsymbol{n} \\
d
\end{array}\right)=\left(\begin{array}{l}
0 \\
0 \\
0
\end{array}\right)
$$

with the additional constraint that $\|\boldsymbol{n}\|=1$. 
Therefore, the squared distance between point $\mathbf{p}$ and the plane containing $f$ is

$$
Q^{f}(\mathbf{p})=\left(\boldsymbol{n}^{T} \mathbf{p}+d\right)^{2}=\mathbf{p}^{T}\left(\boldsymbol{n} \boldsymbol{n}^{T}\right) \mathbf{p}+2 d \boldsymbol{n}^{T} \mathbf{p}+d^{2},
$$

which can be represented as a quadric functional $\mathbf{p}^{T} \mathbf{A p}+2 \mathbf{b}^{T} \mathbf{p}+c$, where $\mathbf{A}$ is a symmetric $3 \times 3$ matrix, $\mathbf{b}$ is a column vector of size 3 , and $c$ is a scalar. Thus,

$$
Q^{f}=(\mathbf{A}, \mathbf{b}, c)=\left(\boldsymbol{n} \boldsymbol{n}^{T}, d \boldsymbol{n}, d^{2}\right)
$$

which can be stored using 10 coefficients $(6+3+1)$. The advantage of this representation is that the quadric is obtained as a simple linear combination of these coefficient vectors.

Finally, a quadric for the distance error metric of a new vertex $v$ after an edge collapse $\left(v_{1}, v_{2}\right) \rightarrow v$ is formulated as the sum of the quadrics on its adjacent faces, weighted by face area for vertex $v_{1}$ and $v_{2}$ :

$$
Q^{v}=\sum_{v_{1} \in f} \operatorname{area}(f) \cdot Q^{f}+\sum_{v_{2} \in f} \operatorname{area}(f) \cdot Q^{f} .
$$

\subsection{Feature Edge Error Metric}

We extend Lindstrom's approach [1] to reconstructing the feature lines of an original model by preserving surface boundaries. A feature error metric is defined to be the distance between the plane of the associated feature edge $e_{f e}$ and a point. We define the normal to the feature edge $e_{f e}$ by averaging the normals of two neighboring triangles that share that edge. We then construct a plane $m_{e_{1}}$ which is close to orthogonal to the edge. This plane is spanned by the edge $e_{1}^{n}=\left(n_{1}^{f}+n_{2}^{f}\right) / 2$, and $m_{e_{1}}=e_{1} \times e_{1}^{n}$, where $e_{1}^{n}$ is the computed normal at the edge $e_{1}$ (see Fig. 11).

Using these estimates, we define the feature edge error metric for feature edge $e_{f e}$ of a vertex as the sum of the squared distances from a point $v$ to the planes containing the neighboring feature edges:

$$
Q^{f e}=\sum_{e \in f e(v)}\left(m_{e}\right)\left(m_{e}\right)^{T} .
$$

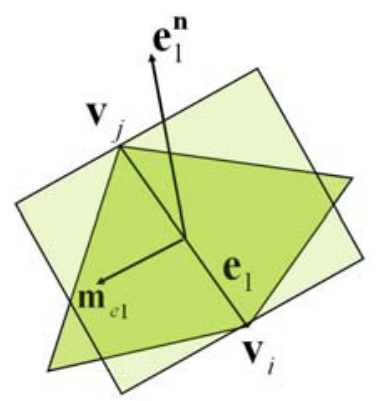

Fig. 1. Computation of feature edge error metric 


\section{Simplification Algorithm}

Our simplification algorithm is based on iterative edge collapses using an error metric which is a combination of the surface error and the feature edge error, based on geometric correspondence in 3D. The error metric is defined as the sum:

$$
Q^{v}=\sum_{v_{n} \in n g b h(v)} Q^{v_{n}}+\sum_{f e_{n} \in f e(v)} Q^{f e_{n}} .
$$

where the geometric error $Q^{v_{n}}$ is the squared distance from a vertex on the plane $\mathbf{v} \subset \mathbf{R}^{3}$ containing the incident triangle, and the feature edge error $Q_{f e_{n}}$ is the distance between a point and orthogonal vector calculated from edge $e_{f e}$. We start with a polygonal model and perform a simplification algorithm. For each edge collapse $\left(v_{1}, v_{2}\right) \rightarrow \bar{v}$, the simplification algorithm determines the error of $v$ and then moves $v$ to a new position that minimizes the error. The edge collapse with the lowest error is chosen and carried out. The algorithm can be summarized as follows:

Algorithm: The simplification algorithm.

Step 1 Extract ridge and valley lines.

Step 2 Compute the new quadrics for the initial polygonal model using Eq. (3).

Step 3 Compute the new vertex position for each edge collapse $\left(v_{1}, v_{2}\right) \rightarrow \bar{v}$. The error metric in the portion of the vertex $v$ represents the cost of that collapse.

Step 4 Place all the edge collapses in a heap with the minimum-cost edge at the top.

Step 5 Iteratively remove the edge collapse of least cost from the heap, collapse this edge, and update the costs of all edge collapses involving $v_{1}$ and $v_{2}$.

\section{Implementation and Results}

All models were simplified on a PC with a Pentium IV 3.0Ghz processor and $1 \mathrm{~GB}$ of main memory. We simplified a number of models, and used MeshDev 12, 15, to measure the surface deviation after simplification. The MeshDev software [1] compares two triangular meshes according to geometrical data or appearance attributes. It returns visual and numerical results. The method is based on the geometric error used by Metro tool [2].

Figs. 2 show the mean geometric deviations between the original and the simplified models. There is the comparison with four graphs representing geometric errors of simplified models using with feature edge and without it. It can be seen from these graphs that the simplified meshes generated using our algorithm have smaller mean geometric errors than meshes produced using without feature error metrics. In Figs. 3 and 4, our method preserves the silhouettes of the back and teeth more accurately.

Fig. 5 shows various degrees of simplification. When simplified to 700 faces without feature error quadrics, the model lost one propeller. But our simplified result did not lose a propeller until the model was simplified to 536 faces. 


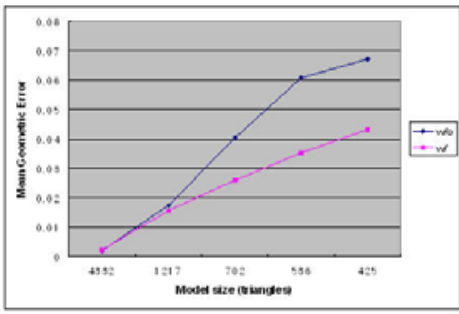

(a)

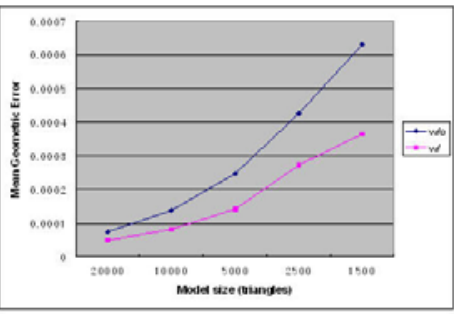

(b)

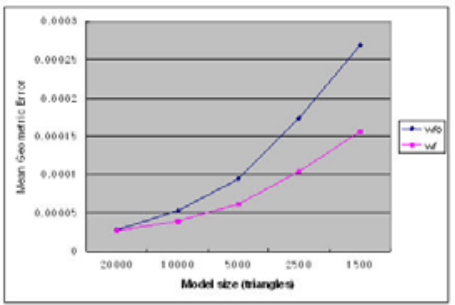

(c)

Fig. 2. Mean geometric error measured by MeshDev [1]: (a) Cessna, (b) Dragon and (c) Dinosaur model. $w /$ :with feature edge error metric, $w / o$ : without feature edge error metric.

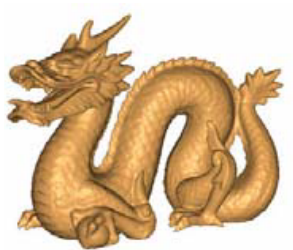

(a)

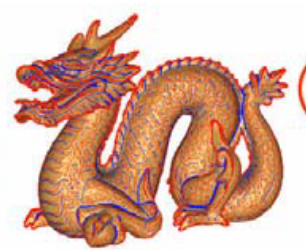

(b)

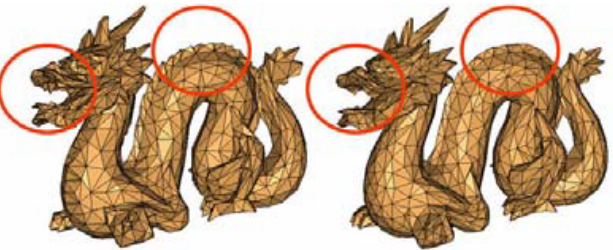

(c) (d)

Fig. 3. Simplified results for the Dragon model. (a) original model (320,000 faces), (b) red ridges and blue valleys, c) with feature edge error metric, (d) without feature edge error metric.

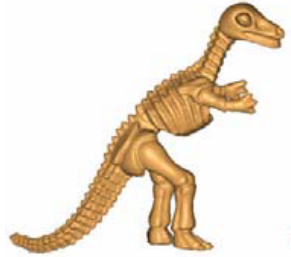

(a)

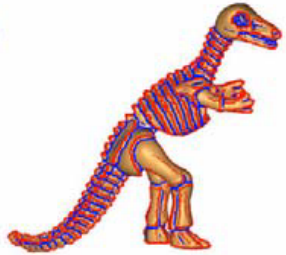

(b)

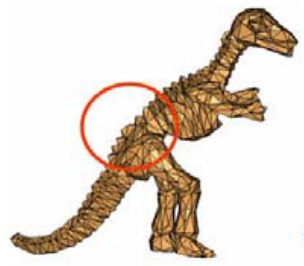

(c)

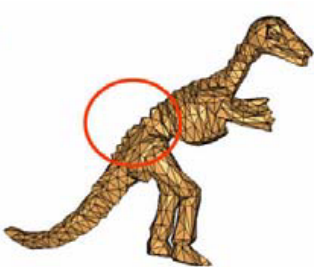

(d)

Fig. 4. Simplified results for the Dinosaur model. (a) original model (112,623 faces), (b) red ridges and blue valleys, c) with feature edge error metric, (d) without feature edge error metric. 


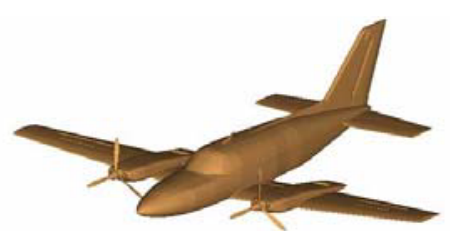

(a)

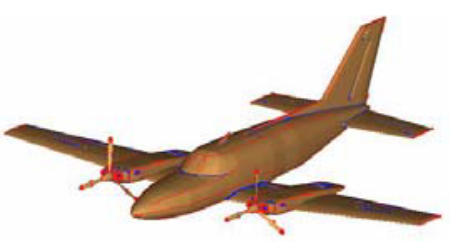

(b)

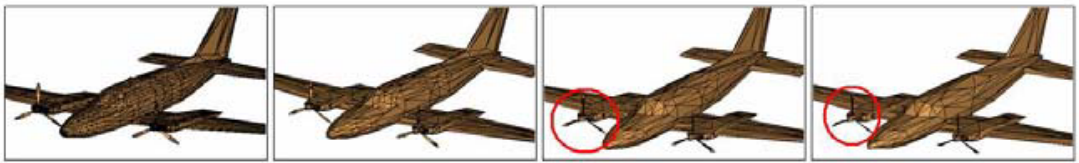

(c)

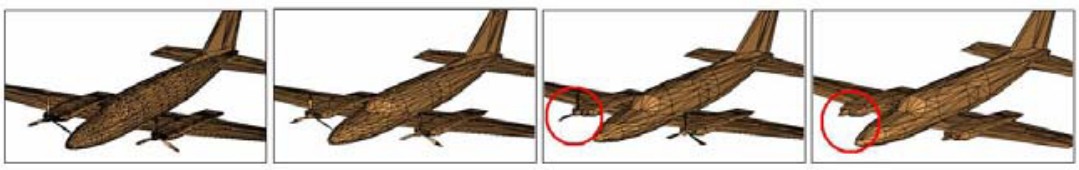

(d)

Fig. 5. Simplified results of the Cessna model. (a) original model (13,546 faces), (b) red ridges and blue valleys, (c) and (d) are each simplified to 4332, 1217, 702, and 536 faces.

\section{Conclusion and Future Work}

We have proposed a method of simplifying polyhedral models which extracts and preserves salient features. Simplification is controlled by a feature error metric combined with a basic error metric. The new error metric overcomes the drawback of a basic error metric which is inaccurate on highly curved regions, and preserves sharp or prominent regions despite a drastic simplification.

In future work, it would be desirable to measure the error metrics of attributes for surface simplification and provide a more theoretical analysis.

Acknowledgments. This work was supported by grant No.(R01-2005-00010120-0) from Korea Science and Engineering Foundation in Ministry of Science \& Technology, and Three-Dimensional Digital Real Object Duplication System (RODS) Development Project from the ministry of commerce, industry and energy. This research was also supported by University IT Research Center Project.

\section{References}

1. http://meshdev.sourceforge.net/

2. Cignoni P., Rocchini C., and Scopigno R.: Metro: measuring error on simplification surfaces. Computer Graphics Forum, 17(2):167-174, 1998.

3. Cohen J., Olano M., and Manocha D.: Appearance-Preserving Simplification. In Proceedings of SIGGRAPH 98, pp.115-122, 1998. 
4. Garland M. and Heckbert P. S.: Surface simplification using quadric error metrics. In Proceedings of SIGGRAPH 97, pp.209-216, 1991.

5. Garland M. and Heckbert P. S.: Simplifying surfaces with color and texture using quadric error metrics. In Proceedings of IEEE Visualization 98, pp.263-269, 1998.

6. Hoppe H.: New Quadric Metric for simplifying Meshes with Appearance Attributes. In Proceedings of IEEE Visualization, pp.59-66, 1999.

7. Hubeli A. and Gross M.: Multiresolution Feature Extraction from Unstructured Meshes. In Proceedings of IEEE Visualization 01, 2001.

8. Kim S.-J., Kim S.-K. and Kim C.-H.: Discrete Differential Error Metric For Surface Simplification. In Proceedings of Pacific Graphics 2002, pp.276-283, October 2002.

9. Kim S.-K. and Kim C.-H.: Finding Ridges and Valleys in a Discrete Surface Using a Modified MLS Approximation. Computer-Aided Design, 37(14):1533-1542, 2005.

10. Kho Y. and Garland M.: User-Guided Simplification. In Proceedings of ACM Symposium on Interactive 3D Graphics. 2003.

11. Lindstrom P. and Silva C. T.: A Memory Insensitive Technique for Large Model Simplification. In Proceedings of IEEE Visualization, pp.121-126, 2001.

12. Luebke D., Reddy M., Cohen J., Varshney A., Watson B., and R. Huebner: Level of Detail for 3D Graphics. Morgan Kaufmann, 2003.

13. Pojar E. and Schmalstieg D.: User-controlled creation of multiresolution meshes. In Proceedings of ACM Symposium on Interactive 3D Graphics, pp.127-130, 2003.

14. Rossignac J. and Borrel P.: Multi-resolution 3D approximations for rendering complex scenes. Modeling in Computer Graphics: Methods and Application, pp.455465, 1993.

15. Roy, M., Foufou, S., and Truchetet, F. Mesh Comparison using Attribute Deviation Metric. International Journal of Image and Graphics (IJIG), 4(1):127-140, 2004.

16. Schroeder, W. J., Zarge, J. A, and Lorensen, W. E. Decimation of Triangle Meshes. Computer Graphics (In Proceedings of SIGGRAPH 92), 26(2):65-70, 1992.

17. Watanabe, K., and Belyaev, A. G. Detection of Salient Curvature Features on Polygonal Surfaces. Computer Graphics Forum (In Proceedings of Eurographics 01), 20(3):385-392, 2001.

18. Walter, J. D., and Healey, C. G. Attribute Preserving Dataset Simplification. In Proceedings of IEEE Visualization 01, pp.113-120, 2001.

19. Yoshizawa, S., Belyaev, A. G., and Seidel, H.-P. Fast and robust detection of crest lines on meshes. In Proceedings of ACM Symposium on Solid and Physical Modeling, pp.227-232, 2005. 